\title{
El parto pretérmino desde los registros del nacido vivo en Colombia, período $2008-2017$
}

\author{
Néstor Ricardo Gerónimo Ortega a \\ ${ }^{a}$ Médico, especialista en gerencia hospitalaria, Magister en Epidemiología. Grupo Salud Materna y Perinatal, Subdirección de Investigación Científica y \\ Tecnológica, Dirección de Investigación en Salud Pública, Instituto Nacional de Salud, Colombia. \\ ORCID: https://orcid.org/0000-0003-3855-9267,ngeronimo@ins.gov.co \\ DOI: https://doi.org/10.22517/25395203.22091
}

\section{Resumen.}

Introducción: El parto pretérmino es uno de los principales problemas de la salud infantil. Y la principal causa de mortalidad infantil en los países en vía de desarrollo. En los últimos 20 años ha venido en aumento en el mundo por diferentes causas, conocer su tendencia y características poblacionales es un elemento útil para su atención.

Objetivo: Describir las características poblacionales asociadas al parto pretérmino en Colombia desde los datos del Registro del Nacido Vivo en la década 2008 -2017 .

Métodos: Estudio descriptivo ecológico a partir de fuentes segundarias: microdatos de Estadísticas Vitales del DANE; información almacenada en el programa Excel de Microsoft Office ${ }^{\circledR}$. Análisis de las variables tiempo/lugar/persona en el programa estadístico SPSS ${ }^{\mathrm{TM}}$ mediante estadística descriptiva, análisis de asociación utilizando el estadístico Ji-cuadrado y el coeficiente de correlación Phi para la asociación estadística entre variables.

Resultados. Fueron analizados 6.705 .385 registros de nacidos vivos, 1.277 .839 correspondientes a parto hasta las 37 semanas de gestación. Prevalencia del 19\% de todos los años. Departamentos con mayor tasa de prematuros: Atlántico, Bogotá, Antioquía y Santander. No se encontró una asociación fuerte con los datos del registro del nacido vivo.

Conclusiones: Existe déficit de información acerca del parto pretérmino en aspectos de datos completos y accesibles a los investigadores. La información no es homogénea ni sigue parámetros específicos. El registro de nacido vivo, sin embargo, es un excelente instrumento de recolección de datos poblacionales y base de aproximación al evento.

Palabrasclave:Partopretermino;reciénnacidoprematuro; estudios ecológicos; Coheficiente de correlación de Phi.
Preterm birth from live-born registry in the period between 2008 - 2017 in Colombia

\section{Abstract}

Background: Preterm birth is one of the main problems in infant health and it is the leading cause of infant mortality in developing countries. During the last 20 years this particularity has been increasing in the world for different reasons. Knowing its trend and population characteristics is a useful element for its attention.

Objectives: To describe the population characteristics associated with preterm birth in Colombia from the data of the Live Birth Registry in the decade 2008 - 2017.

Methods: Descriptive ecological study using secondary sources such us Vital Statistics microdata from DANE; information stored in the Microsoft Office ${ }^{\circledR}$ Excel program. Also, the analysis of the time/place/person variables in the SPSS TM statistical program through descriptive statistics, and association analysis using the Chi-square statistic and the Phi correlation coefficient for the statistical association between variables was implemented.

Results: $6,705,385$ records from live-born registry were analyzed. $1,277,839$ corresponded to preterm birth up to 37 weeks of gestation with $19 \%$ of prevalence all years. The departments with the highest rate of premature infants were Atlántico, Bogotá, Antioquia, and Santander. No strong association was found with live birth registry data.

Conclusions: There is lack of complete and accessible information to researchers related to preterm birth. The data is not homogeneous and does not follow specific parameters. However, the live-born registry is an excellent instrument for collecting population data and a useful basis for approaching this event.

Keywords: Premature delivery; Ecological Studies; Phi correlation coefficient, premature newborn. 


\section{Introducción}

Según la definición de la Organización Mundial de la Salud-OMS, el parto prematuro o pretérmino es aquel que ocurre antes de las 37 semanas de gestación $(1,2)$. Existen varios factores de riesgo conocidos que llevan a que ocurra un parto pretérmino, como lo es la edad de la gestante, problemas de salud: hipertensión, tabaquismo, diabetes, los cuidados de la materna o los problemas que desarrolla durante la gestación $(3,4)$ in order to recognize high risk women and to provide a global overview of the Italian situation. Study design: A multicenter, observational and retrospective, cross-sectional study was designed. The study population comprised 7634 women recruited in 9 different University Maternity Hospitals in Italy. The main criteria for inclusion were: women having had vaginal preterm or term spontaneous delivery in each participating centre during the study period. The records related to deliveries occurring between April and December 2008. A multivariable logistic regression was employed to identify independent predictors of spontaneous preterm birth. Odds ratios (ORs. La salud infantil es una de las prioridades de salud y desarrollo en todas las regiones del mundo, la muerte prematura de niños y niñas en los primeros años de vida continúa siendo una de las problemáticas en salud con mayores requerimientos de acciones desde todos los sectores, convirtiéndose así en una meta para el desarrollo y el bienestar de las comunidades $(5,6)$. Según la OMS nacen niños prematuros en países de ingresos altos, medianos y bajos; cada año nacen unos 15 millones de niños prematuros, es decir más de 1 de cada 10; el 60\% de ellos nace en el África subsahariana y en Asia meridional(1).

Disminuir la mortalidad infantil por causas prevenibles es uno de los objetivos planteados en las políticas de salud del país, sin embargo, este continúa siendo una problemática de salud relacionada a su vez con factores sociales, económicos, culturales, políticos y ambientales que inciden para determinar la forma en la que las personas se enferman y mueren. Analizar los factores asociados al parto pretérmino cobra relevancia en la prevención y reducción de la mortalidad neonatal e infantil en el país, dada su relación con diversos factores de tipo socioeconómico, demográficos, de morbilidad materna y durante la gestación(7-9).

Se estima que cada año nacen unos 15 millones de niños prematuros (antes de que se cumplan las 37 semanas de gestación). Esa cifra está aumentando en el mundo, según la Organización Mundial de la Salud - OMS. Aproximadamente un millón de niños prematuros mueren cada año debido a complicaciones en el parto(10)the Millennium Development Goal (MDG. Muchos de los bebés prematuros que sobreviven sufren algún tipo de discapacidad de por vida, en particular, discapacidades relacionadas con el aprendizaje y problemas visuales y auditivos(11-13)en la provincia de Buenos Aires, y se los cita posteriormente a un control kinésico mensual. Se utilizó como método de evaluación las pautas o jalones madurativos correspondientes al bebé normal nacido a término. Se registraron aquellos bebés, tanto prematuros como de término, que mostraron atrasos en el desarrollo, ingresándolos a tratamiento de estimulación motriz y se registró su evolución. El tratamiento kinésico se basó en los métodos de neurodesarrollo (NDT. Esto problemas ponen en riesgo la vida o integridad de los recién nacidos y deben pasar un proceso de adaptación, muchas veces con finales inesperados. Al proceso de transición se suman la inmadurez de órganos y funciones vitales (pulmón, tracto gastrointestinal, termorregulación, riñón, etc.), la fragilidad de sistemas corporales y las condiciones asociadas o desencadenantes del parto prematuro (por ejemplo, insuficiencia placentaria, ruptura prematura de membranas, corioamnionitis, etc. $(2,14,15)$ Entre las causas más frecuentes del parto prematuro figuran los embarazos múltiples, las infecciones y las enfermedades crónicas, la diabetes y la hipertensión; aunque, a menudo no se identifica la causa.

En Colombia contamos con sistemas de información acerca de las llamadas estadísticas vitales - EEVV (nacimientos y defunciones) que permiten hacer una aproximación del comportamiento poblacional del fenómeno del parto prematuro. Una de esas fuentes es las bases de microdatos anonimizados del DANE, que nos permitirá ver el comportamiento del parto pretérmino en una década, para tratar de determinar asociaciones entre los datos demográficos de la madre y los desenlaces entre la población de nacidos vivos.

\section{Métodos}

Se trata de un estudio observacional, de tipo ecológico de estudio de grupos múltiples(16), con datos de Estadísticas Vitales del Departamento Administrativo Nacional de Estadística - DANE. Los datos son de dominio público, de carácter oficial, desde el enlace de microdatos anonimizados de defunciones y nacimientos(17). Los datos fueron descargados al equipo de cómputo del Instituto Nacional de Salud segregado por años, desde 2008 a 2017, donde fueron depurados, haciendo coincidencia en la metricidad y parámetros de las variables, así como la unión de los archivos en uno solo, para su análisis en paquete estadístico. Se recodificaron nuevas variables para un mejor análisis, se cambiaron en algunas ocasiones la parametrización de algunas y se ajustó a la probabilidad de análisis.

El trabajo de alistamiento de la base de datos, así como la revisión de otras fuentes, permitió evidenciar que había algunos problemas de los datos según la metodología utilizada por el DANE y la utilizada oficialmente por el sector salud en Colombia. Los principales hallazgos 
fueron:los datos DANE y Ministerio de Salud y Protección Social - MSPS, coinciden en cantidad total, mas no en los grupos de edad gestacional.

La definición de parto pretérmino es: "el nacimiento se produce antes de completarse la semana 37 de gestación. El feto crece y madura en la vida intrauterina a un ritmo predecible y biológicamente determinado. Al completar la semana 37 de gestación suele estar lo suficientemente maduro y con suficiente tamaño corporal como para hacer una transición apropiada a la vida extrauterina." (Guía de práctica clínica del Recién Nacido Prematuro, 2013, MSPS, página 22)

Al hacer las comparaciones de los datos obtenidos se encontró que los grupos poblacionales del DANE incluían a la semana 37 dentro de los grupos de edad pretérmino (Tabla1):

Tabla 1. Semanas de gestación.

\begin{tabular}{|c|c|c|c|}
\hline DANE & FRECUENCIAS & MSPS - SISPRO & FRECUENCIAS \\
\hline Menos de 22 & 735 & 1 - MENOS DE 22 SEMANAS & 560 \\
\hline De 22 a 27 & 24.703 & 2 - DE 22 A 27 SEMANAS & 19.689 \\
\hline De 28 a 37 & 1.252 .401 & 3 - DE 28 A 36 SEMANAS & 467.145 \\
\hline De 38 a 41 & 5.351 .195 & 4 - DE 37 A 41 SEMANAS & 4.757 .263 \\
\hline De 42 y más & 26.670 & 5 - DE 42 O MÁS SEMANAS & 17.460 \\
\hline Ignorado & 15.685 & NO APLICA & 6.789 \\
\hline Sin información & 33.996 & SIN INFORMACIÓN & 1.436 .479 \\
\hline TOTAL & 6.705 .385 & TOTAL & 6.705 .385 \\
\hline
\end{tabular}

Además, se debía tener en cuenta que los datos SISPRO - MSPS son datos agrupados, no individualizados, que no se pueden descargar de manera específica por casos, al contrario de lo que sucede de los microdatos de DANE, utilizados en este análisis. También se encontró que ninguna de las dos bases de datos permite realizar el análisis de las diferentes subcategorías del Parto Pretérmino, propuesta por la Organización Mundial de la Salud, al solo encontrarse datos agrupados, no individualizados.

Así las cosas, se decidió realizar el análisis de la base de datos del DANE, por su practicidad y posibilidad de análisis bioestadístico, dejando la salvedad de la inclusión de la semana 37 dentro de los partos pretérmino. No se hizo criterio de inclusión, sino que se procesaron los 6.705.385 registros, correspondiente al periodo de 2008 a 2017 . No se esperaban duplicados, ya que se trata de una información de carácter oficial. Las bases se integraron en una sola y se procesó en bloque.

Los datos resultados de las variables definidas fueron procesados mediante el software SPSS (Statistical Package for the Social Sciences) versión 22, utilizado para realizar la captura y análisis de datos. Se crearon nuevas variables en combinación, en caso de que sean necesarias en el análisis descriptivo de las mismas. El análisis descriptivo se hizo según la naturaleza de cada variable. Posteriormente, se realizó otro análisis descriptivo del parto prematuro univariado, para, posteriormente, terminar con un análisis multivariado, utilizando el estadístico Ji-cuadrado y el coeficiente de correlación Phi para analizar la asociación estadística entre variables y la magnitud y dirección de su relación. En el caso de la Ji-cuadrado se establece que con una $\mathrm{p}>0,05$ se aceptará la hipótesis nula y no habrá asociación estadísticamente significativa. La magnitud del coeficiente de correlación Phi se estableció como baja si está en el rango de 0,3 a 0,5 y alta su supera el 0,5 hasta 1 . La direccionalidad se dará por el signo positivo o negativo del mismo. Todos y cada uno de los cuadros y gráficas del presente artículo son de producción propia y con única fuente en la base de datos DANE referida anteriormente.

\section{Resultados}

En Colombia, en la mencionada década, hubo un total de 6.705.385 nacimientos, con 1.277.839 partos pretérmino, en promedio, el 19\%, distribuidos en cada año así (Tabla 2): 
Tabla 2. Distribución de partos pretérmino en el período 2008-2017

\begin{tabular}{|l|c|c|c|c|c|c|c|c|c|c|c|}
\hline & $\mathbf{2 0 0 8}$ & $\mathbf{2 0 0 9}$ & $\mathbf{2 0 1 0}$ & $\mathbf{2 0 1 1}$ & $\mathbf{2 0 1 2}$ & $\mathbf{2 0 1 3}$ & $\mathbf{2 0 1 4}$ & $\mathbf{2 0 1 5}$ & $\mathbf{2 0 1 6}$ & $\mathbf{2 0 1 7}$ & TOTAL \\
NACIMIENTOS & 715.453 & 699.775 & 654.627 & 665.499 & 676.835 & 658.835 & 669.137 & 660.999 & 647.521 & 656.704 & 6.705 .385 \\
\hline N. PRETERMINO & 131.091 & 126.775 & 118.862 & 122.544 & 128.017 & 127.410 & 130.385 & 130.186 & 130.092 & 132.477 & 1.277 .839 \\
\hline $\begin{array}{l}\text { TASA BRUTA } \\
\text { PREMATUREZ } \\
\text { X 100 NV }\end{array}$ & 18,3 & 18,1 & 18,2 & 18,4 & 18,9 & 19,3 & 19,5 & 19,7 & 20,1 & 20,2 & $\begin{array}{c}19,0 \\
\text { PROME- } \\
\text { DIO }\end{array}$ \\
\hline
\end{tabular}

La tendencia general de nacimientos fue a la baja, con una diferencia entre 2008 y 2017 de 58.749, que representa una disminución del 8,21\% (Figura 1):

Figura 1. Tendencias de nacimientos en Colombia, período 2008-2017

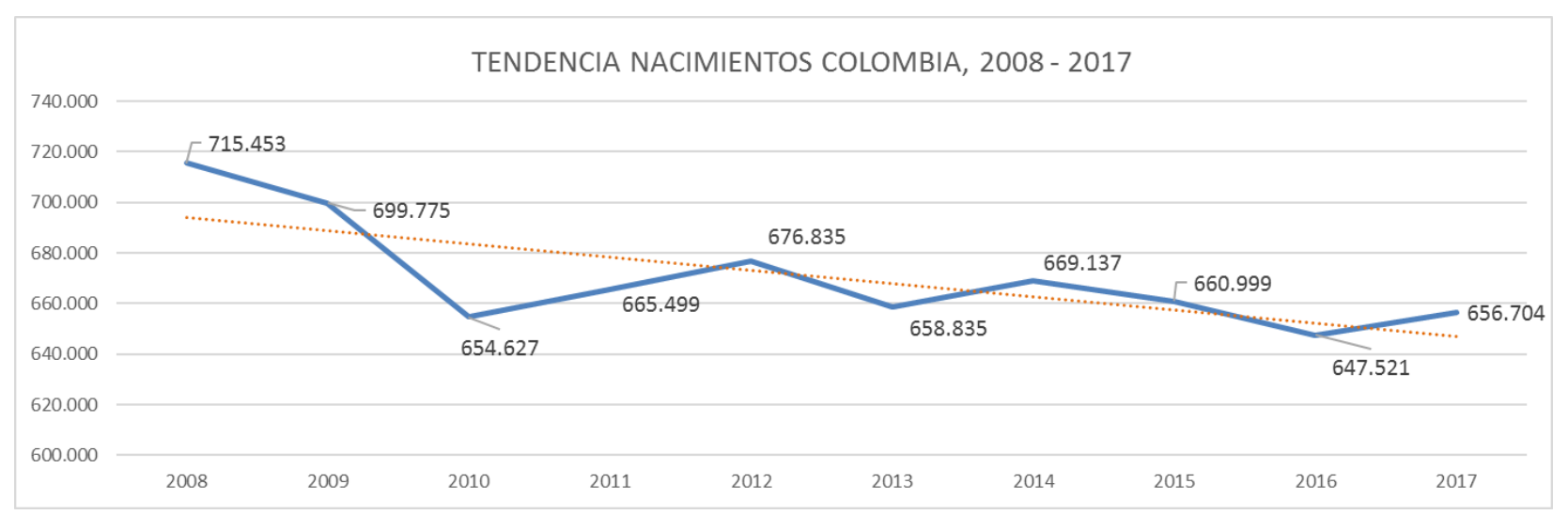

Los meses con más nacimientos, en todos los años, son septiembre, octubre y agosto, en orden de importancia, representando el 9,1\%, 8,6\% y 8,6\%, respectivamente, del número de nacimientos en todos los años de la década.

Sin embargo, la tasa bruta de natalidad (X 1.000 hab.) no ha variado tanto, como muestra la Figura 2:

Figura 2. Tasa bruta de natalidad en Colombia, período 2008-2017

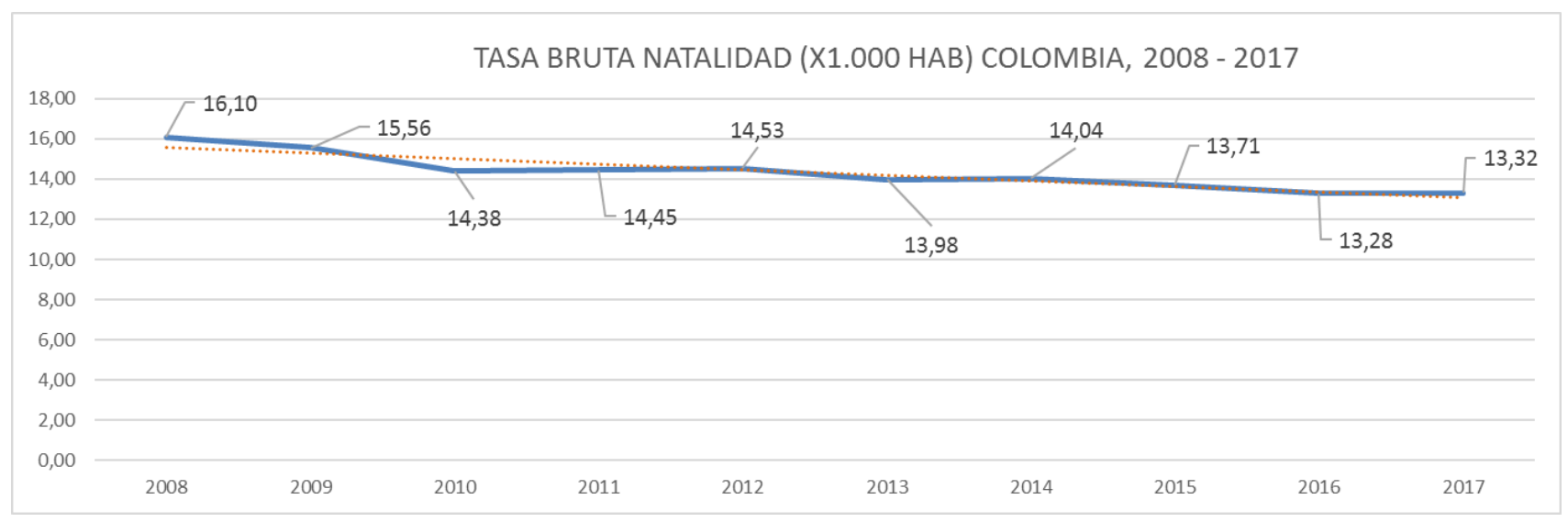

De esta manera, el primer año del período tenía una tasa bruta de natalidad de 16,10 nacimientos por cada mil habitantes en el país, terminando en el 2017 con 13,32 X 1.000 hab. 
Los departamentos con más nacimientos, representando el 53,5\%, en la década fueron Bogotá (17,72\%), Antioquia (11,70\%), Valle del Cauca (8,37\%), Atlántico (6,03\%), Bolívar (5\%) y Santander (4,69\%). No se hizo análisis de la frecuencia en municipios debido a la codificación de los mismos, impidiendo su identificación.

La variable sexo fue representada por el masculino, con un 51,39\%, seguido del femenino $(48,61 \%)$ y el indeterminado.

Como se había planteado, se hizo una recodificación de variable semanas de gestación a dicotómica, como se observa en la Tabla 3:

Tabla 3. Recodificación de variable semanas de gestación a dicotómica

\begin{tabular}{|c|c|c|c|c|}
\hline PREMATUREZ & Frecuencia & Porcentaje & $\begin{array}{c}\text { Porcentaje } \\
\text { válido }\end{array}$ & $\begin{array}{c}\text { Porcentaje } \\
\text { acumulado }\end{array}$ \\
\hline HASTA 37 SEM & 1.277 .839 & 19,06 & 19,20 & 19,20 \\
\hline MAYOR A 38 SEM & 5.377 .865 & 80,20 & 80,80 & 100,00 \\
\hline Total & 6.655 .704 & 99,26 & 100,00 & \\
\hline IGNORADO & 15.685 & 0,23 & & \\
\hline SIN INFORMACIÓN & 33.996 & 0,51 & & \\
\hline Total & 49.681 & 0,74 & & \\
\hline TOTAL GENERAL & 6.705 .385 & 100,00 & & \\
\hline
\end{tabular}

Así las cosas, los nacimientos hasta la semana 37 representan el 19,2\% (1.277.839 casos) de todos los nacimientos. Un $0,74 \%$ de los datos no son calculados por carecer de esa información.

La división que hace el DANE de la edad gestacional se muestra en la Tabla 4:

Tabla 4. DANE, División de la edad gestacional.

\begin{tabular}{|l|l|l|l|l|}
\hline EDAD GESTACIONAL & Frecuencia & Porcentaje & Porcentaje válido & $\begin{array}{c}\text { Porcentaje } \\
\text { acumulado }\end{array}$ \\
\hline Menos de 22 & 735 & 0,06 & 0,06 & 0,06 \\
\hline De 22 a 27 & 24.703 & 1,93 & 1,93 & 1,99 \\
\hline De 28 a 37 & 1.252 .401 & 98,01 & 98,01 & 100,00 \\
\hline Total & 1.277 .839 & 100,00 & 100,00 & \\
\hline
\end{tabular}

Por esto se deduce que el $98 \%$ de los partos pretérmino ocurrieron entre la semana número 28 y la semana número 37 de gestación.

Los departamentos con más nacimientos pretérmino son los mismos que en la población general de nacidos vivos, representando el 58,42\% de todos. Tenemos a Bogotá con el 20,23\% de los nacimientos pretérmino, seguido de Antioquia (12,75\%), Valle del Cauca (8,39\%), Atlántico (7,14\%), Bolívar (4,96\%) y Santander (4,96\%). Sin embargo, al realizar el cálculo de la tasa bruta de prematurez general, (Número de nacimientos pretérmino / Número de nacimientos X cien nacidos vivos), se cambian algunos lugares, así: Atlántico (22,6\%), Bogotá (21,7\%), Antioquía (20,8\%), Santander $(20,1 \%)$ y Valle del Cauca (19,1\%). Los partos pretémino fueron atendidos en centros de prestación de servicios de salud y, por lo tanto, fueron clasificados con sitio de nacimiento en cabecera municipal, con un $99 \%$ en ambas ocasiones.

La clasificación por sexo de los partos pretérmino fue masculino con 52,32\%, seguido de femenino $(47,68 \%)$ e indeterminado $(0,01 \%)$

Respecto al peso, el $63,96 \%$ de los nacimientos prematuros fueron reportados con peso adecuado, mayor de 2.500 gramos. En la talla, el $94,58 \%$ de los nacimientos pretérmino fueron reportadas con talla adecuada al nacer. Para los partos pretérmino, el $45,6 \%$ de ellos se trataba del primer hijo de la mujer y en un $30,8 \%$ de su segundo hijo. El 
comportamiento es similar al de la población general de nacimientos.

Respeto al tipo de parto, los nacidos prematuramente recibieron la clasificación presentada en la Tabla 5:

Tabla 5. Tipos de parto

\begin{tabular}{|l|l|l|l|l|}
\hline \multicolumn{1}{|c|}{ TIPO DE PARTO } & \multicolumn{1}{|c|}{ Frecuencia } & \multicolumn{1}{c|}{ Porcentaje } & Porcentaje válido & Porcentaje acumulado \\
\hline Espontáneo & 574.518 & 44,96 & 45,00 & 45,00 \\
\hline Cesárea & 691.234 & 54,09 & 54,14 & 99,14 \\
\hline Instrumentado & 10.791 & 0,84 & 0,85 & 99,99 \\
\hline Ignorado & 125 & 0,01 & 0,01 & 100,00 \\
\hline Total & 1.276 .668 & 99,91 & 100,00 & \\
\hline Sin información & 1.171 & 0,09 & & \\
\hline & 1.277 .839 & 100,00 & & \\
\hline
\end{tabular}

En cuanto a la multiplicidad del embarazo, el 92,4\% de los partos pretérmino fueron en casos de embarazo simple, único.

La edad de la madre al momento del parto, en los partos hasta las 37 semanas tuvo el comportamiento que se muestra en la Tabla 6:

Tabla 6. Edad de la madre

\begin{tabular}{|c|c|c|c|c|}
\hline EDAD DE LA MADRE & Frecuencia & Porcentaje & Porcentaje válido & $\begin{array}{c}\text { Porcentaje } \\
\text { acumulado }\end{array}$ \\
\hline De 10-14 Años & 16.132 & 1,26 & 1,26 & 1,26 \\
\hline De 15-19 Años & 283.208 & 22,16 & 22,16 & 23,43 \\
\hline De 20-24 Años & 352.312 & 27,57 & 27,57 & 51,00 \\
\hline De 25-29 Años & 277.438 & 21,71 & 21,71 & 72,71 \\
\hline De 30-34 Años & 199.431 & 15,61 & 15,61 & 88,31 \\
\hline De 35-39 Años & 111.296 & 8,71 & 8,71 & 97,02 \\
\hline De 40-44 Años & 32.518 & 2,54 & 2,54 & 99,57 \\
\hline De 45-49 Años & 3.011 & 0,24 & 0,24 & 99,81 \\
\hline De 50-54 Años & 2.491 & 0,19 & 0,19 & 100,00 \\
\hline Total & 1.277 .837 & 100,00 & 100,00 & \\
\hline Sin información & 2 & 0,00 & & \\
\hline
\end{tabular}

Aquí se presenta un aumento del porcentaje de mujeres en el rango entre 15 y 19 años, así como los de 25 a 29 años, respecto al comportamiento de la población general de nacimientos.

El estado civil de la mujer al momento del parto, para los nacimientos pretérmino se muestra en la Tabla 7 : 
Tabla 7. Estado civil de la madre en parto pretérmino.

\begin{tabular}{|c|c|c|c|c|}
\hline ESTADO CIVIL MADRE & Frecuencia & Porcentaje & $\begin{array}{c}\text { Porcentaje } \\
\text { válido }\end{array}$ & $\begin{array}{c}\text { Porcentaje } \\
\text { acumulado }\end{array}$ \\
\hline $\begin{array}{c}\text { No está casada y lleva dos o más años } \\
\text { viviendo con su pareja }\end{array}$ & 560.583 & 43,87 & 44,79 & 44,79 \\
\hline $\begin{array}{c}\text { No está casada y lleva menos de dos años } \\
\text { viviendo con su pareja }\end{array}$ & 261.270 & 20,45 & 20,87 & 65,66 \\
\hline Está separada, divorciada & 6.270 & 0,49 & 0,50 & 66,16 \\
\hline Está viuda & 1.934 & 0,15 & 0,15 & 66,32 \\
\hline Está soltera & 196.736 & 15,40 & 15,72 & 82,03 \\
\hline Está casada & 224.894 & 17,60 & 17,97 & 100,00 \\
\hline Total & 1.251 .687 & 97,95 & 100,00 & \\
\hline Sin información & 26.152 & 2,05 & & \\
\hline
\end{tabular}

Aquí, se mantiene el porcentaje de mujeres viviendo en Unión libre con pareja como mayoría, pero para el parto pretérmino se aumenta el porcentaje de la madre soltera durante el evento.

Para el análisis de las condiciones demográficas y sociales del nacimiento y el parto pretérmino en Colombia, en el período 2008 - 2017, se hizo confrontación de variables categóricas por medio de tablas de contingencia, utilizando el estadístico Ji-cuadrado, y el coeficiente de correlación Phi. Los valores de la variable Prematurez son los recodificados de "Hasta 37 semanas" y "Mayor de 38 semanas"

A pesar de que en las pruebas estadísticas, hubo muy poco resultado apreciable, principalmente porque aunque el JiCuadrado se presenta como con asociación en todos los resultados, principalmente por el número elevado de medidas que se llevan a cabo, el coeficiente de correlación Phi nos advertía de una relación sumamente baja, muchísimo menor a 0,5 . En la Tabla 8 se presentan las asociaciones: 
Tabla 8. Asociaciones entre la prematurez y distintos valores

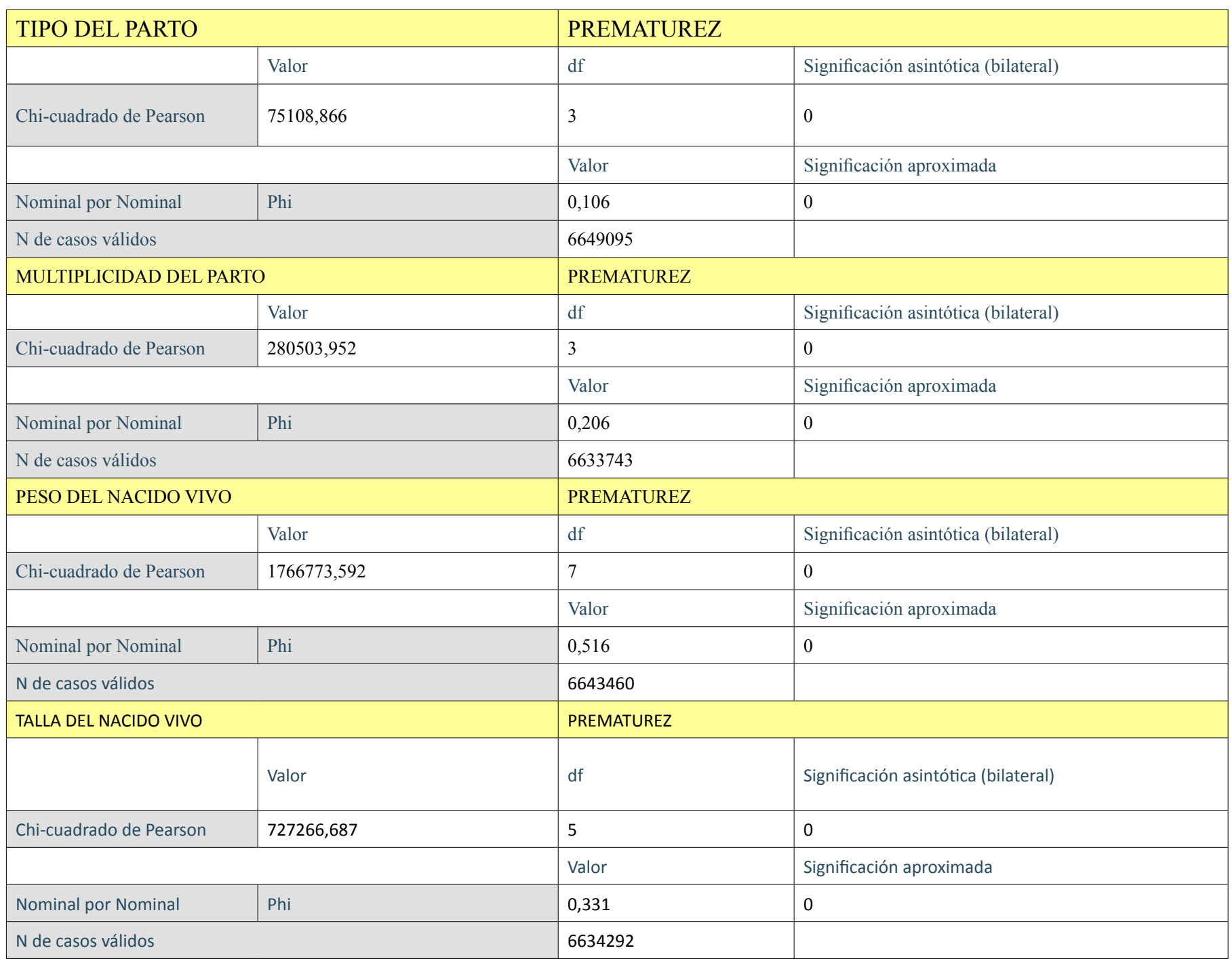

Según estos datos, existe una relación entre el tipo del parto y el evento de parto pretérmino, muy baja, directamente proporcional; la relación de cesáreas cambia entre parto a término y el pretérmino mientras que la resolución del embarazo es mayor por cesárea en un parto pretérmino. Igualmente, se evidencia una asociación entre la multiparidad en el embarazo y el parto pretérmino y una asociación entre el peso del nacido vivo y el parto pretérmino, asociación fuerte y directamente proporcional. Además, se presenta una asociación entre la talla del nacido vivo y el parto pretérmino, asociación baja y directamente proporcional.

\section{Discusión}

Existe déficit de información acerca del parto pretérmino en aspectos de datos completos y accesibles a los investigadores. La información no es homogénea ni sigue parámetros específicos. El registro de nacido vivo, sin embargo, es un excelente instrumento de recolección de datos poblacionales y base de aproximación al evento (18). Desde 2008 el país ha adaptado y rediseñado el mejoramiento tecnológico del Sistema de Registro Civil y Estadísticas Vitales, acerca de la gestión en tiempo real de los registros de nacidos vivos, lo que mejoraba notablemente el acceso a los datos. Sin embargo, los datos individualizados y anonimizados del DANE tienen agrupaciones y puntos de corte distintas a las usadas en el sector salud, lo que crea una deficiencia en el análisis de la información.

Los principales departamentos del país ven reflejado su fenómeno poblacional en la representatividad de los nacimientos y los nacimientos pretérmino. Sin embargo, las tasas brutas de prematurez por departamento dan una mayor carga al departamento del Atlántico sobre Bogotá y Antioquia. Este fenómeno es digno de ser investigado más minuciosamente. 
Aproximadamente el $19 \%$ de todos los nacimientos son pretérmino y el comportamiento se refleja en todos los años estudiados en el decenio. Resulta interesante ver que los datos de peso y talla no corresponden a lo caracterizado como de parto pretérmino, ya que se encuentra mucho dato de peso y talla adecuados. Esto podría ser un nuevo proyecto de investigación.

La edad de la madre no parece tener una influencia en la probabilidad de tener un parto pretérmino, tal como se comporta como la población general de nacidos vivos, así como tampoco lo es el estado civil de la madre.

Tratando de hacer una correlación entre los datos del certificado de nacido vivo y el parto pretérmino se utilizó indicadores de asociación y coeficiente de correlación. El uso del coeficiente correlación de Phi nos permitió descartar la asociación que parece darnos el Ji-cuadrado. Las únicas variables que presentaron algún resultado para apreciar fueron las de tipo de parto, multiparidad de este embarazo, el peso y la talla del recién nacido vivo. Aun así, parece natural esta asociación, puesto que la resolución quirúrgica (cesárea) para el parto pretérmino tiene mayor representatividad, pues es uno de las grandes estrategias para garantizar sobrevida del recién nacido pretérmino, como lo afirman diversos autores(19-21). De igual manera, relacionar el peso y la talla para un parto pretérmino es evidente debido al retraso del crecimiento esperado.

De esta manera, es prudente recomendar que se haga un ajuste a la información contenida en los microdatos del DANE, para afinar la clasificación y agrupación del evento parto pretérmino, así como poder calcular los cortes adecuados para manejar las clasificaciones de la Organización Mundial de la Salud. También corresponde plantear otro estudio epidemiológico de relaciones específicas de riesgo, tipo casos y controles, de ser posible.

\section{Referencias}

1. Organización Mundial de la Salud. Nacidos Demasiado Pronto. Informe de Acción Global sobre Nacimientos Prematuros [Internet] 2012 [Consultado 2020 Sep 14] Disponible en: https://www.who.int/maternal_child adolescent/documents/born_too_soon/es/

2. Ministerio de Salud y Proteccion Social. Guía de práctica clínica del recién nacido prematuro [Internet] 2013 [Consultado 2020 Sep 9]Disponible en: https:// www.minsalud.gov.co/sites/rid/Lists/BibliotecaDigital/ RIDE/INEC/IETS/GPC_Completa_Premat.pdf

3. Di Renzo GC, Giardina I, Rosati A, Clerici G, Torricelli M, Petraglia F. Maternal risk factors for preterm birth: A country-based population analysis. Eur J Obstet Gynecol Reprod Biol. 2011 Dec 1;159(2):342-6.

4. Alberto Villanueva Egan L, Karina Contreras Gutiérrez A, Pichardo Cuevas M, Rosales Lucio J. Perfil epidemiológico del parto prematuro. Rev Chil Obstet Ginecol. 2008; 09:542-8.

5. Ministerio de Salud y Protección Social. Plan Decenal de Salud Pública 2012-2021 [Internet] 2012 [Consultado 2019 Sep 14] Disponible en: https://www.minsalud.gov. $\mathrm{co} / \mathrm{sites} / \mathrm{rid} /$ Lists/BibliotecaDigital/RIDE/VS/ED/PSP/ PDSP.pdf

6. Ministerio de Salud y Protección Social C. Plan Nacional de Salud Pública 2007-2010 [Internet] 2007 [Consultado 2019 Sep 14] Disponible en: https://www.paho.org/col/index.php?option=com d o c m a n \& vi e w = down lo ad \& c a t e g ory slug $=$ publicaciones-ops-oms-colombia $\&$ alias $=1194-$ plan-nacional-salud-publica-2007-10\&Itemid $=688$

7. Instituto Nacional de Salud. Mortalidad Evitable en Colombia para 1998-2011 [Internet]. 2014 [Consultado 2020 Sep 14] Disponible en: https://www.ins.gov.co/ Direcciones/ONS/Informes/3. Mortalidad evitable.pdf

8. Hernández A, Rodríguez- Hernández J, Cubillos -Novella A, Santacruz -Caicedo M, Vega-Romero R y García -Becerra A. Determinantes sociales de las desigualdades en mortalidad materna y neonatal en las comunidades indígenas arhuaca y wayuu.Pontificia Universidad Javeriana. 2017.

9. Jaramillo-Mejía MC, Chernichovsky D, JiménezMoleón JJ. Determinantes de la mortalidad infantil en Colombia. Path Análisis Determinants of infant mortality in Colombia. Path Analysis. Rev Salud Pública. 2018;20(1):3-9.

10. Liu L, Oza S, Hogan D, Chu Y, Perin J, Zhu J, et al. Global, regional, and national causes of under-5 
mortality in 2000-15: an updated systematic analysis with implications for the Sustainable Development Goals. Lancet. 2016;388(10063):3027-35.

11. Claudia Mauro, M. Villegas de Martínez, M. A. Berselini, T. Calarco. Bebés prematuros y recién nacidos de término de riesgo: su evaluación, seguimiento y propuestas de servicio. Rev Iberoam Fisioter y Kinesiol. 2001;4(1):2-7.

12. Bello M, Rovira M, Juanet J, Mas MM, Subira MM, Ribera Vilella T. Los bebés prematuros y su desarrollo visual. Rev sobre Discapac Vis. 2010;58:1-5.

13. Jansson-Verkasalo E, Haverinen S, Valkama AM, Korpilahti P. Los niños prematuros tienen un alto riesgo de dificultades en el procesamiento auditivo central, evidenciadas mediante los potenciales evocados auditivos. Rev Logop Foniatr y Audiol. 2011 Jul $1 ; 31(3): 125-32$.

14. Ministerio de Salud y Proteccion Social. Guía de práctica clínica del recién nacido prematuro [Internet]. Vol. 2. 2013 [Consultado 2019 Sep 14]. p. 2-70. Disponible en: https:/www.minsalud.gov.co/sites/ $\mathrm{rid} /$ Lists/BibliotecaDigital/RIDE/INEC/IETS/GPC_ Completa_Premat.pdf

15. Escobar-Padilla B, Gordillo-Lara LD, MartínezPuon H. Risk factors associated with preterm birth in a second level hospital. Rev Med Inst Mex Seguro Soc. 2017;55(4):424-8.

16. Álvaro Sepúlveda M, Stephanie Kobrich S, Rodolfo Guiñez G, Jorge Hasbun H. Morbidity of late preterm: Current evidence and new approach. Rev Chil Obstet Ginecol. 2012;77(2):154-8.

17. Borja-Aburto VH. Estudios ecológicos. Salud Publica Mex. 2000;42(6):533-8.

18. Departamento Administrativo de Estadistica - DANE C. Microdatos estadísticas vitales, DANE, Colombia. 2019.

19. Ministerio de Salud y Protección Social de Colombia. Certificado de nacimiento [Internet] 1993 [Consultado 2019 Sep 14]. Disponible en: https://nd.ruaf.gov.co/ WebSiteNDE/BirthsPages/CertificadoNacimiento.aspx

20. Fuchs K, Wapner R. Elective Cesarean Section and Induction and Their Impact on Late Preterm Births. Clin Perinatol. 2006 Dec;33(4):793-801.

21. Barros FC, Vélez MDP. Temporal trends of preterm birth subtypes and neonatal outcomes. Obstet Gynecol. 2006 May;107(5):1035-41.
22. Eduardo A. Valenti, Diego Enriquez, Miguel Larguía. Parto pretermino provocado. Rev del Hosp Matern Infant Ramón Sardá. 2000;19(2):72-7. 